\title{
L'acte De Parole Perlocutoire Dans Le Film Sur La Piste Du Marsupilami D'alain Chabat
}

Khilyatul Aulia ${ }^{\bowtie}$ Sri Rejeki Urip ${ }^{\bowtie}$ Anastasia Pudjitriherwanti

Département de la Langue et la Littérature Étrangère, Faculté des Langues et des Arts, Universitas Negeri Semarang, Indonesia

\section{Info d'article}

Histoire de l'Atrticle :

Reçu Janvier 2020

Accepté Mars 2020

Publié Mai 2020

Keywords :

perlocutionary,

Marsupilami.

\begin{abstract}
Each utterance pronounced by the speakers has certain meaning and certain objectives in accordance with their respective objective. Austin distinguishes three types of speech acts, these are locutionary, illocutionary and perlocutionary. The aim of the research is to know (1) what functions of the perlocutionary speech acts in the film On The Track of Marsupilami and (2) what effects of the perlocutionary speech acts containing in the dialogue of the film On the track of Marsupilami. The methods and techniques of data acquisition are the methods of observation with the basic technique of tapping and advanced techniques of SLBC (listen without participating) and note. The data were analyzed with the Padan method and the basic Sorting of Certain Elements (PUP) technique, then continue with the Relate, Compare and Assimilate (HBS) techniques and then Connect and Compare the Principle (HBSP). The method of presenting the result of the data analysis used is the informal method with the technique of presenting the data used of ordinary words. Based on the analysis, I found effects of perlocutionary acts which are the intended effect and the unintended effect, there are 42 perlocutionary acts with expected effects and 13 perlocutionary acts with unintended effects. There is only one declarative perlocutionary act with the intended effect, 10 representative perlocutoires with the intended effects and 7 of unforeseen perlocutoires, 3 of expressive perlocutoires with the intended effects, 4 of unexpected perlocutoires, 24 directive perlocutoires with the intended effects and 1 with the unforeseen effect, 3 comissive perlocutoires with the expected effects and 1 with the unforeseen effect.
\end{abstract}

\section{Extrait}

Chaque énoncée prononcée par les locuteurs a certaine signification et certains objectifs conformément à leur objectif respectifs. Austin distingue trois types d'actes de langage, ce sont locutoire, illocutoire et perlocutoire. Le but de la recherche est savoir (1) quelles fonctions des actes de parole perlocutoire dans le film Sur La Piste de Marsupilami et (2) quels effets des actes de parole perlocutoire contenant dans le dialogue du film Sur la piste de Marsupilami. Les méthodes et les techniques d'acquisition de données sont les méthodes d'observation avec la technique de base de la tapotement et de techniques avancées de SLBC (écouter sans participer) et noter. Les données ont été analysées avec la methode de Padan et la technique de base Trier des Certains Élements (PUP), puis continuer avec les techniques Relier, Comparer et assimiler (HBS) et puis Connecter et Comparer la Principe (HBSP). La méthode de présentation du résultat de l'analyse des données utilisée est la méthode informelle avec la technique de présentation des données utilisées des mots ordinaires. Basé sur l'analyse, j'ai trouvé des effets d'actes perlocutoires qui sont l'effets prévus et l'effet imprévus, il y a 42 actes perlocutoires avec des effets prévus et 13 actes perlocutoires avec des effets imprévus. Il y a seulement un acte perlocutoire déclaratif avec l'effet prévu, 10 perlocutoires representatives avec les effets prévus et 7 de perlocutoires imprévus, 3 de perlocutoires expressives avec les effets prévus, 4 de perlocutoire imprévus, 24 perlocutoires directive avec les effets prévu et 1 avec l'effet imprévu, 3 perlocutoires comissives avec les effets prévus et 1 avec l'effet imprévu.

(C) 2020 Universitas Negeri Semarang 


\section{INTRODUCTION}

La langue est une moyenne utilisée dans les conversations (Chaer 2007: 53). Une communication se déroulera sans problème si la langue qui est utilisée est correcte. Cela signifie que la langue est utilisée conformément à la situation et à la condition du locuteur et que la nature du discours est mise en œuvre. Cela dépend des déterminants du langage ou de la communication, c'est-à-dire la réglage ou la scene, le participant, le fin ou le destination, l'acte, la clé, l'instruments, la norme, et les genres.. Chaque énoncé qui est prononcé par le locuteur a une certaine signification et un certain but. La parole dans les discours sont deux choses qui se produisent dans un processus de communication. les actes de langage ont une variété de types, dont l'un est le regroupement basé sur la nature de la relation dans laquelle comprend locutoire, ilocutoire, et perlocutoire. Selon l'opinion de Yule (2006: 82), les actes de langage sont les actions affichées par le discours.

Perlocutoire est un discours qui vise à créer un effet sur allocutaire de sorte que le partenaire de la parole soit affectée par le discours de locuteur. L'effet peut être causé intentionnellement ou involontairement par le haut-parleur (Rustono 1999: 37).

Dans cette étude, la chercheuse sont intéressé par le choix du film Sur La Piste $\mathrm{Du}$ Marsupilami d'Alain Chabat comme objet de recherche. la chercheuse voit que dans le film, de nombreuses conversations sont des actes de perlocutoire.

\section{MÉTHODE DE RECHERCHE}

L'approche utilisée dans cette recherche est l'approche qualitative. Moloeng (2007: 4) cite la notion de Bogdan et Taylor (1975: 5) qui mentionne que la méthodologie qualitative comme procédure de recherche produit des données descriptives sous forme de mots écrits ou oraux de personnes et de comportements observables.

La méthode utilisée dans cette recherche est une recherche qualitative descriptive, c'est-à-dire une recherche qui décrit des données sous forme de phrases, de mots et non de nombres. Une approche descriptive a été choisie car les données sous la forme d'un discours dans le film Sur la Piste du Marsupilami d'Alain Chabat doivent être décrites pour expliquer les fonctions, les effets et les aspects de la perlocution contenue dans le film.

Les données collectionnes dans cette étude sont les discours contenus dans le film Sur La Piste de Marsupilami qui contient du perlocutoire. Les données sont ensuite analysées en fonction des fonctions, des effets et des aspects associés à l'effet de la parole perlocutoire.

La source de données utilisée dans cette recherche est un film intitulé Sur La Piste de Marsupilami réalisé et écrit par Alain Chabat. Le film a été lancé le 4 avril 2012 avec une durée de 105 minutes.

Les méthodes et les techniques d'acquisition de données sont la méthode d'observation avec la technique de base de la tapotement et de techniques avancées de SLBC (écouter sans participer) et noter. Les données ont été analysées avec la methode de Padan et la technique de base Trier des Certains Élements (PUP), puis continuer avec les techniques Relier, Comparer et Assimiler (HBS) et puis Connecter et Comparer la Principe (HBSP). La méthode de présentation du résultat de l'analyse des données utilisée est la méthode informelle avec la technique de présentation des données utilisées des mots ordinaires.

\section{RÉSULTAT ET DISCUSSION}

\section{Fonction Déclarative}

Les acte perlocutoire avec la fonction déclarative est la parole avec l'intention de légitimer, décider, annuler, interdire, permettre, accorder, soulever, classifier, et pardonner. Dans cette étude, la chercheuse ont trouvé un seul discours qui est l'acte de perlocutoire avec fonction déclarative. L'action de la perlocutoire déclarative se trouve dans la dialogue suivante: 
(1) Contexte: Ce discours se produit après que le chef de Paya ait dit la prophétie Chixculub. La prophétie a expliqué que Pablito et Dan Geraldo étaient les personnes choisies qui pouvaient sauver la Paya de la destruction.

Le Chèf du Paya : Vous êtes les élus de la prophétie de Chicxulub.

Les Payas : Chicxulub!

Le caractère gras dans le dialogue (1) est un type de discours déclaré l'acte de perlocutoire avec un effet prévu. La déclaration appartient au discours déclaratif perlocutoire car il décide que Dan Geraldo et Pablito sont les élus de la prévision du Chicxulube. L'effet de la danse tarifaire tribale est que tous les gens de Paya triomphent pour saluer en appelant à "Chixculub!".

\section{Fonction Représentant}

L'act de la perlocutoire avec fonction représentative est un discours qui est un énoncé de faits, d'affirmations, de conclusions et de descriptions. Dans cette étude, la chercheuse ont trouvé 17 actes de langage qui sont un acte de la perlocutoire représentative. L'acte de la perlocutoire représentatif se trouve dans le dialogue suivant:

(2) Contexte : Les conversations se déroulent entre Cassandra avec deux touristes sur le bord de la route. Cassandra qui a fait semblant de pleurer son perroquet alors il a fait venir les deux touristes et l'aider.

Le tourist : Qu'est-ce qui se passe ma chéri?

Cassandra : : Mon perroquet est malade, il va mourir.

Le peroquet : Ca va pas du tout.

La touriste : tul'as pas emmené là?

Cassandra: J'ai pas d'argent.

Dans le dialogue (2) il y a un acte de langage qui est un acte de la perlocutoire sur la phrase en gras. Cet acte a une fonction représentative avec l'effet prévu. Il est dit être un acte de discours de discours représentatif parce que le discours se plaint. L'effet qui est résulté est les touristes se sentent pitié et disposés à aider à apporter la perroquet pour le traitement.

\section{Fonction Expressive}

Les discours de perlocution avec des fonctions expressives sont des actes de langage qui reflètent des déclarations psychologiques et peuvent être des expressions de joie, de difficulté, de joie, de haine, de plaisir ou de misère. Dans cette étude, les auteurs ont trouvé seulement sept discours qui sont des actes de langage perlocutoire avec la fonction expressive. Voici une boîte de dialogue qui contient des actes de la perlocutoire expressifs:

(3) Contexte : Le déguisement général comme Céline Dion et chante d'une voix très mélodieuse pour distraire les soldats gardant son palais. Le général a chanté pour signaler Dan Dan Geraldo et Pablito pour entrer dans la cour de sauvetage de Marsupilami.

Guerrier : Allez, vous venez! C'est énorme!

Le dialogue (19) est un type d'actes de langage exprimés perlocutoire avec l'effet prévu. On dit que c'est un acte de langage perlocutoire expressif parce que le discours est une déclaration de joie. Les effets résultant de ce discours des soldats qui étaient de garde à l'intérieur du palais sont sortis du palais pour regarder Céline Dion chanter.

\section{Fonction Directive}

L'acte de parole perlocutoire avec la fonction directive de ce type de discours reconnait ce que le locuteur veut. Dans cette étude, les auteurs ont trouvé jusqu'à 25 discours qui sont des actes de langage perlokusi avec fonction directive. Voici une boîte de dialogue qui contient des actes de langage perlokusi avec fonction directive.

(21) Contexte: Des soldats s'approchent de Dan Geraldo pour exécuter les ordres du général d'amener Dan Geraldo à son palais. Mais parce que Dan a refusé, le soldat l'électrocutait de sorte que Dan Geraldo était impuissant et l'a pris.

Le soldat : Le général Pochero vent vous voir.

Dan : Non, en fait parce que moi, j'ai rendez-vous avec Pablito Camaron.

Le soldat : Il m'a fatigué avec ses explications. Allez.

Le caractère gras dans le dialogue (26) est un acte de la perlocutoire directive avec l'effet prévu. On dit que c'est un acte de langage perlocutoire directive parce que le discours est 
commandant. La raillerie a produit un effet sur les autres soldats, ils ont rapidement amené Dan Geraldo à sa voiture au palais.

\section{Fonction Commissive}

L'acte de discours perlocutoire avec fonction commissive est un acte qui lie son locuteur à effectuer ce qui est mentionné dans son discours. Les types d'actes de langage inclus dans ce type d'acte de langage sont les accords, les menaces, les dénégations et les garanties. Dans cette étude, les auteurs ont trouvé seulement cinq discours qui sont des actes de la perlokutoire avec la fonction commissive. Voici un dialogue qui contient de la perlocutoire commissive.

(51) Contexte: Mathéo est venu chez Pablito pour recouvrer des dettes. Pablito n'a pas pu payer ses dettes, laissant Mathéo et ses gardes du corps en colère et le menaçant.

Pablito : Ecoutez. Je vais te rembourses, je te jure Mathéo, je te paierai. Laisse-moi un peu de temps, j'ai un plan.

Discours dans le dialogue (51) est un acte de discours de la perlocutoire commissive avec l'effet prévu. On dit que c'est un acte de la perlocutoire commissif parce que le discours est prometteur. Après avoir écouté le discours de Pablito, Mathéo et son garde du corps étaient prêts à lui donner le temps de rembourser sa dette, même s'il devait libérer son perroquet.

\section{CONCLUSION}

Sur la base de l'analyse des perlocutoire suivi dans le film travail Sur La Piste d'Alain Chabat Marsupilami qui a été décrit dans le chapitre IV, on peut conclure que:

Dans le film Sur La Piste de Marsupilami d'Alain Chabat, les auteurs ont trouvé 54 discours qui sont des actes de la perlocutoire. L'auteur a trouvé 1 perlocutoire déclaratif, 17 perlocutoires représentantives, 7 perlocutoires expressifs, 25 perlocutoires directives et 4 perlocutoires commisives.

Sur la base de l'analyse effectuée sur le film Sur la piste du Marsupilami, il y a des effets résultant d'actes de perlocutoire sous forme de titres prévus et les effets imprévus. Il y a 41 actes de la perlocutoire avec des effets prévus et 13 actes de la perlocutoire avec des effets imprévus. 1 perlocutoire déclarative avec l'effet prévu, 10 actes de perlocutoires rèpresantantives avec des effets prévus, 7 actes de perlocutoires rèpresantantives avec des effets imprévus, 3 actes de perlocutoires expressives avec des effets prévus, 4 actes de perlocutoires expressives avec des effets imprévus, 24 actes de perlocutoires directives avec des effets prévus, 1 acte de perlocutoire directive avec d'effets prévu, 3 actes de perlocutoires commissives avec des effets prévus, et 1 actes de perlocutoires commisives avec des effets imprévus.

Les actes de la perlocutoire les plus dominants dans le film Sur la Piste du Marsupilami est un acte de la perlocutoire directive avec l'effet planifiés. il y a 25 données qui est l'acte de la perlocutoire directive de toutes les 54 données. L'effet qui domine l'acte de perlokusi dans le film est l'effet planifié, avec un total de 35 sur 54 données.

\section{BIBLIOGRAPHIE}

Austin, J.L. 1962. How To Do Things With Words. London : Oxford University Press.

Chaer, Abdul. 2007. Kajian Bahasa, Jakarta, Rineka Cipta.

Fallou, MBOW. 2011. "L'acte de requete dans l'interaction verbale". Revue Electronique Internationale de Science du Langage Sudlangues. Desember 2011. Nomor 16:105-121. Senegal:Université Cheik Anta DIOP.

Ghosh, Srinjoy. 2016. "Explorations Of The Perlocutionary”. English Studies International Research Journal. Volume 4 Issue 2 (2016)

Hakim, Arif Rahman. 2010. Tindak Tutur Perlokusi pada Komik Kilonjy.com. Semarang. Unnes.

Kusumaningsih, Indah Apriyanti. 2016. Tindak Tutur Ilokusi dalam Film Hors de Prix karya Pierre Salvadori. Yogyakarta: UNY

Leech, Geoffrey. 1983. The Principles of Pragmatics. London: Longman Group Limited.

Moleong, J. L. 2007. Metodologi Penelitian Kualitatif:Edisi Revisi. Bandung: PT. Remaja.

Nadar, F. X. 2009. Pragmatik dan Penelitia Pragmatik. Yogyakarta: Graha Ilmu. 
Nurhayati, Wahyu A. Dwi \& Yuwartatik. 2016. Illocutionary and Perlocutionary Acts on Main Characters Dialogues in John Milne's Novel: The Black Cat. IJOLTL (2016), 1(1): 67-96.

Parera, J.D. 2004. Teori Semantik. Jakarta: Erlangga.

Riyanto, Subur. 2015. Tindak Tutur dalam Iklan Radio di Kota Kebumen. Semarang: Unnes.

Rustono, 1999. Pokok-Pokok Pragmatik. Semarang: CV IKIP SEMARANG PRESS.

Sudaryanto. 1993. Metode dan Aneka Teknik Penelitian Bahasa. Yogyakarta: Wacana University Press.

Tarigan, Henry Guntur. 2015. Pengajaran Pragmatik. Bandung: Angkasa.

Yule, George. 2006. Pragmatik. Yogyakarta: Pustaka Pelajar. 